\title{
On strong duality in linear copositive programming *
}

\author{
Kostyukova O.I. ${ }^{\dagger} \quad$ Tchemisova T.V. ${ }^{\ddagger}$
}

\begin{abstract}
The paper is dedicated to the study of strong duality for a problem of linear copositive programming. Based on the recently introduced concept of the set of normalized immobile indices, an extended dual problem is deduced. The dual problem satisfies the strong duality relations and does not require any additional regularity assumptions such as constraint qualifications. The main difference with the previously obtained results consists in the fact that now the extended dual problem uses neither the immobile indices themselves nor the explicit information about the convex hull of these indices.

The strong duality formulations presented in the paper have similar structure and properties as that proposed in the works of M. Ramana, L. Tuncel, and H. Wolkovicz, for semidefinite programming, but are obtained using different techniques.
\end{abstract}

Key words. Linear Copositive Programming, strong duality, normalized immobile index set, extended dual problem, Constraint Qualifications, Semi-infinite Programming (SIP), Semidefinite programming (SDP)

AMS subject classification. 90C25, 90C30, 90C34

\section{Introduction}

Linear Copositive Programming problems can be considered as linear programs over the convex cone of so-called copositive matrices (i.e. matrices which are positive semi-defined on the nonnegative orthant). Copositive problems form a special class of conic optimization problems and have many important applications, including $\mathcal{N} \mathcal{P}$-hard problems. For the references on applications of Copositive Programming see e.g. [5, 8] and others.

Linear copositive problems are closely related to that of linear Semi-Infinite Programming (SIP) and Semidefinite Programming (SDP). Linear copositive and semidefinite problems are particular cases of SIP problems but Linear Copositive Programming deals with more challenging and less studied problems than that of SDP. The literature on theory and methods of SIP, SDP and Linear Copositive Programming is very interesting and quite large, we refer the interested readers to $[5,6,8,11,18,19,24,25,28]$, and the references therein.

*This work was partially supported state research program "Convergence"(Republic Belarus), Task 1.3.01 and by Portuguese funds through CIDMA - Center for Research and Development in Mathematics and Applications, and FCT - Portuguese Foundation for Science and Technology, within the project UID/MAT/04106/2019.

${ }^{\dagger}$ Institute of Mathematics, National Academy of Sciences of Belarus, Surganov str. 11, 220072, Minsk, Belarus (kostyukova@im.bas-net.by).

${ }_{\ddagger}^{\ddagger}$ Mathematical Department, University of Aveiro, Campus Universitario Santiago, 3810-193, Aveiro, Portugal (tatiana@mat.ua.pt). 
Although the concepts of co-positivity and complete positivity were originally formulated in 1952 in the paper by T. Motzkin [21], an active research in theory and methods of Linear Copositive Programming has begun only in the recent decades in papers of I. Bomze, M, Dür, E. de Klerk, and others (see [4, 5, 7]).

Optimality conditions and the associated duality relationships are among the central topics of convex optimization and the importance of their study is well recognized (see e.g. [6, 26], and the references therein). Optimality conditions are a crucial issue in the study of any optimization problem since they allow not only to test the optimality of a given feasible solution, but also to develop efficient numerical methods. As it is mentioned in [19], the duality plays a central role in detecting infeasibility, lower-bounding of the optimal objective value, as well as in design and analysis of iterative algorithms.

Often the studies on optimality conditions and duality for finite and (semi-) infinite programming use certain regularity assumptions, so-called constraint qualifications (CQ). Such assumptions permit to guarantee in some particular cases a strong (or zero-gap) duality which means that the optimal values of the primal and dual objective functions are equal and, hence, the difference between these values (the duality gap) vanishes.

It is a known fact that in Linear Programming (LP), the strong duality is guaranteed without any CQ ([6]). The duality results for LP can be generalized to some particular classes of optimization problems. Several attempts were done to obtain CQ-free optimality and strong duality results for different classes of convex SIP problems (see e.g. $[10,12,14]$ ).

In $[25,27]$, a CQ-free duality theory for conic optimization was developed in terms of so-called minimal cone. Being quite general, this theory has one disadvantage in terms of its application, namely, it is very abstract. In a number of publications, various explicit dual formulations were obtained by applying this theory to SDP problems (see e.g. [11, 24, 26, 27]) and other optimization problems over symmetric (i.e., self-dual and homogeneous) cones (see [23]). As it was mentioned in [22, 23], finding a broader family of conic problems for which such explicit dual formulations are possible, is an open problem.

Linear copositive problems belong to a wider and more complex class of linear conic problems than that of SDP, namely, to the class of optimization problems over cones of copositive and $\backslash$ or completely positive matrices that are neither self-dual nor homogeneous (see [9]). The duality theory for these problems is not well studied yet. It is worth to mention that almost all duality results and optimality conditions for Linear Copositive Programming are formulated under the Slater CQ ([1, 4]).

In our papers $[12,14,15]$, and others, we developed a new approach to optimality in SIP and SDP. This approach is based on the notion of immobile indices of constraints of an optimization problem, which refers to the indices of the constraints that are active for all feasible solutions. In $[13,16]$, we have applied our approach to problems of Linear Copositive Programming and successfully obtained new explicit CQ-free optimality conditions and strong duality results. It is essential that to formulate our results, we used either the immobile indices ([16]), or the vertices of the convex hull of the normalized immobile index set ([13]).

In this paper, we further develop our approach to linear copositive problems and use it to obtain a new dual problem which we refer to here as the extended dual problem. As well as the regularized dual problem from [13], the extended dual one is constructed using the notion and properties of the normalized immobile indices, but in the formulation of this problem neither these indices nor the vertices of the convex hull of the corresponding index set are present. This permitted us to formulate the extended dual problem for linear copositive problem in an explicit form and avoid the use of additional procedures for finding the immobile indices. The 
new extended dual problem satisfies the strong duality relations without any CQ.

One interesting property of the obtained results consists in the fact that the new dual formulations for Linear Copositive Programming are closely related to the dual problems proposed in $[24,25,27]$ for SDP. This relation not only confirms the already known deep connection between copositive and semidefinite problems but, taking into account the impact of the duality results of M. Ramana et al. in relation to SDP, permits one to expect that the proposed here duality results are also very promising in Linear Copositive Programming.

It is worth to mention here that at present, with exception of [13], there are no explicit strong duality formulations without CQs for Linear Copositive Progamming. All the results presented in the paper are original and cannot be obtained as a direct extension of any previous results.

The paper is organized as follows. Section 1 hosts Introduction. In section 2, given a linear copositive problem, we formulate the corresponding normalized immobile index set and establish some new properties of this set. An extended dual problem is formulated in section 3 . We prove here that the strong duality property is satisfied. In section 4, we compare the obtained duality results with that presented in [24, 25, 27] for SDP. It is shown that the compared dual formulations for Linear Copositive Programming and SDP are similar, being both CQ-free and providing strong duality. Although these dual formulations were obtained using different techniques, they almost coincide being applied to the class of linear SDP problems. The final section 5 contains some conclusions.

\section{Linear copositive programming problem}

Here and in what follows, we use the following notations. Given an integer $p>1, \mathbb{R}_{+}^{p}$ denotes the set of all $p$ vectors with non-negative components, $\mathcal{S}(p)$ stays for the space of real symmetric $p \times p$ matrices, $\mathcal{P}(p)$ for the cone of symmetric positive semidefinite $p \times p$ matrices, and $\mathcal{C O}^{p}$ for the cone of symmetric copositive $p \times p$ matrices

$$
\mathcal{C O} \mathcal{P}^{p}:=\left\{D \in \mathcal{S}(p): t^{\top} D t \geq 0 \forall t \in \mathbb{R}_{+}^{p}\right\} .
$$

The space $\mathcal{S}(p)$ is considered here as a vector space with the trace inner product:

$$
A \bullet B:=\operatorname{trace}(A B), \text { for } A, B \in \mathcal{S}(p) \text {. }
$$

Consider a linear Copositive Programming problem in the form

$$
\min _{x} c^{\top} x \text { s.t. } \mathcal{A}(x) \in \mathcal{C O} \mathcal{P}^{p},
$$

where the decision variable is $n$-vector $x=\left(x_{1}, \ldots, x_{n}\right)^{\top}$ and the constraints matrix function $\mathcal{A}(x)$ is defined as

$$
\mathcal{A}(x):=\sum_{i=1}^{n} A_{i} x_{i}+A_{0},
$$

matrices $A_{i} \in \mathcal{S}(p), i=0,1, \ldots, n$, and vector $c \in \mathbb{R}^{n}$ are given. Problem (2) can be rewritten as follows:

$$
\min _{x} c^{\top} x \text { s.t. } t^{\top} \mathcal{A}(x) t \geq 0 \forall t \in \mathbb{R}_{+}^{p} .
$$

It is well known that the copositive problem (3) is equivalent to the following convex SIP problem:

$$
\min _{x} c^{\top} x \quad \text { s.t. } t^{\top} \mathcal{A}(x) t \geq 0 \quad \forall t \in T,
$$


with a $p$ - dimensional compact index set in the form of a simplex

$$
T=\left\{t \in \mathbb{R}_{+}^{p}: \mathbf{e}^{\top} t=1\right\},
$$

where $\mathbf{e}=(1,1, \ldots, 1)^{\top} \in \mathbb{R}^{p}, t=\left(t_{k}, k \in P\right)^{\top}, P=\{1,2, \ldots, p\}$.

Denote by $X$ the set of feasible solutions of problems (2) - (4),

$$
X:=\left\{x \in \mathbb{R}^{n}: t^{\top} \mathcal{A}(x) t \geq 0 \quad \forall t \in \mathbb{R}_{+}^{p}\right\}=\left\{x \in \mathbb{R}^{n}: t^{\top} \mathcal{A}(x) t \geq 0 \quad \forall t \in T\right\} .
$$

Evidently, the set $X$ is convex.

According to the definition (see e.g. [16]), the constraints of the SIP problem (4) satisfy the Slater condition if

$$
\exists \bar{x} \in \mathbb{R}^{n} \text { such that } t^{\top} \mathcal{A}(\bar{x}) t>0 \forall t \in T,
$$

and the constraints of the copositive problem (2) satisfy the Slater condition if

$$
\exists \bar{x} \in \mathbb{R}^{n} \text { such that } \mathcal{A}(\bar{x}) \in \operatorname{int} \mathcal{C O} \mathcal{P}^{p}=\left\{D \in \mathcal{S}(p): t^{\top} D t>0 \forall t \in \mathbb{R}_{+}^{p}, t \neq 0\right\} .
$$

Here int $D$ stays for the interior of a set $D$.

Evidently, problems (2), (3), and (4) satisfy or not the Slater condition simultaneously.

Following $[12,13]$, define the sets of immobile indices $T_{i m}$ and $R_{i m}$ in problems (4) and (3), respectively:

$$
T_{i m}:=\left\{t \in T: t^{\top} \mathcal{A}(x) t=0 \quad \forall x \in X\right\}
$$

and

$$
R_{i m}:=\left\{t \in \mathbb{R}_{+}^{p}: t^{\top} \mathcal{A}(x) t=0 \quad \forall x \in X\right\} .
$$

It is evident that the aforementioned sets are interrelated:

$$
R_{i m}=\left\{t \in \mathbb{R}^{p}: t=\alpha \tau, \quad \tau \in T_{i m}, \alpha \geq 0\right\} \text { and } T_{i m}=\left\{t \in R_{i m}: \mathbf{e}^{\top} t=1\right\} .
$$

From the latter relations, we conclude that the set $T_{i m}$, the immobile index set for problem (4), can be considered as a normalized immobile index set for problem (3). In what follows, we will use mainly the set $T_{i m}$, taking into account its relationship with the set $R_{i m}$.

The following proposition is an evident corollary of Proposition 1 from [13].

Proposition 1 Given a linear copositive problem in the form (3), the Slater condition (6) is equivalent to the emptiness of the normalized immobile index set $T_{i m}$.

It is evident that $T_{i m}=\emptyset$ if and only if $R_{i m}=\{\mathbf{0}\}$.

Proposition 2 Given a linear copositive problem (3), let $\{\tau(i), i \in I\}$ be some set consisting of immobile indices of this problem. Then for any $x \in X$, the following inequalities take a place:

$$
\mathcal{A}(x) \tau(i) \geq 0, i \in I \text {. }
$$

The proof of the proposition follows from the definition of immobile indices and Lemma 2.6 from [3]. 
Proposition 3 Given an index set $\{\tau(i) \in T, i \in I\}$, the inequalities

$$
\mathcal{A}(x) \tau(i) \geq 0, i \in I
$$

imply the inequalities

$$
t^{\top} \mathcal{A}(x) t \geq 0 \forall t \in \operatorname{conv}\{\tau(i), i \in I\}
$$

Here conv $S$ denotes the convex hull of a given set $S$.

The proof of the proposition is evident.

Let $\{\tau(i), i \in I\} \subset T_{i m}$ be a nonempty subset of the set of normalized immobile indices in problem (3). For this subset and for any $\varepsilon>0$, denote

$$
\begin{aligned}
& T(\varepsilon):=T(\varepsilon, \tau(i), i \in I):=\{t \in T: \rho(t, \operatorname{conv}\{\tau(i), i \in I\}) \geq \varepsilon\}, \\
& \widehat{T}(\varepsilon):=\widehat{T}(\varepsilon, \tau(i), i \in I):=\{t \in T: \rho(t, \operatorname{conv}\{\tau(i), i \in I\}) \leq \varepsilon\},
\end{aligned}
$$

where $\rho(l, B)=\min _{\tau \in B}|| l-\tau \|$ is the distance between a vector $l$ and a set $B$ associated with the norm $\|a\|=\sqrt{a^{\top} a}$ in the vector space $\mathbb{R}^{p}$. Consider the sets

$$
\mathcal{X}=\left\{x \in \mathbb{R}^{n}: \mathcal{A}(x) \tau(i) \geq 0, i \in I\right\}, \quad \mathcal{X}(\varepsilon)=\left\{z \in \mathcal{X}: t^{\top} \mathcal{A}(z) t \geq 0, \forall t \in T(\varepsilon)\right\} .
$$

The following lemma is a generalization of Lemma 2 from [13].

Lemma 1 Let $\{\tau(i), i \in I\}$ be a subset of the set of normalized immobile indices in problem (3). Then there exists $\varepsilon_{0}>0$ such that $\mathcal{X}\left(\varepsilon_{0}\right)=X$, the set $\mathcal{X}(\varepsilon)$ being defined in (13) with the set $T(\varepsilon)$ as in (11).

Proof. It follows from Proposition 2 that $X \subset \mathcal{X}(\varepsilon)$ for all $\varepsilon>0$. To finalize the proof, it is enough to show that there exists $\varepsilon_{0}>0$ such that $\mathcal{X}\left(\varepsilon_{0}\right) \subset X$. Suppose the contrary. Then for each $\varepsilon>0$ there exist $z(\varepsilon) \in \mathcal{X}(\varepsilon)$ such that

$$
(t(\varepsilon))^{\top} \mathcal{A}(z(\varepsilon)) t(\varepsilon)<0,
$$

where $t(\varepsilon)$ is an optimal solution of the problem

$$
\min _{t \in T} t^{\top} \mathcal{A}(z(\varepsilon)) t
$$

Since, by construction (see Proposition 3 and (13)), it holds

$$
t^{\top} \mathcal{A}(z(\varepsilon)) t \geq 0 \forall t \in T(\varepsilon) \cup \operatorname{conv}\{\tau(i), i \in I\},
$$

then $t(\varepsilon) \in \widehat{T}(\varepsilon) \backslash \operatorname{conv}\{\tau(i), i \in I\}$ for all $\varepsilon>0$. Hence there exists $t^{*}:=\lim _{\varepsilon \rightarrow 0} t(\varepsilon), t^{*} \in$ conv $\{\tau(i), i \in I\}$.

For $\varepsilon>0$, let us consider the vector $l(\varepsilon):=t(\varepsilon)-t^{*}$. By construction, $e^{\top} l(\varepsilon)=0$.

It is evident that there exists a sufficiently small $\bar{\varepsilon}>0$ such that for $k \in P$, the following conditions hold:

$$
\text { if } t_{k}^{*}=0 \text {, then } l_{k}(\bar{\varepsilon})=t_{k}(\bar{\varepsilon}) \geq 0 \text { and if } t_{k}(\bar{\varepsilon})=0 \text {, then } t_{k}^{*}=0 \text { and } l_{k}(\bar{\varepsilon})=0 .
$$


Consequently, the direction $l:=l(\bar{\varepsilon})$ is feasible for $t^{*}$ and $t(\bar{\varepsilon})$ in the set $T$. Hence there exists $\gamma_{0}>1$ such that $t^{*}+\gamma l=t^{*}+\gamma\left(t(\bar{\varepsilon})-t^{*}\right) \geq 0, \mathbf{e}^{\top}\left(t^{*}+\gamma l\right)=1 \quad \forall \gamma \in\left[0, \gamma_{0}\right]$.

Define the function

$$
\begin{aligned}
w(\gamma) & :=\left(t^{*}+\gamma l\right)^{\top} \mathcal{A}(z(\bar{\varepsilon}))\left(t^{*}+\gamma l\right) \\
& =\left(t^{*}\right)^{\top} \mathcal{A}(z(\bar{\varepsilon})) t^{*}+2 \gamma l^{\top} \mathcal{A}(z(\bar{\varepsilon})) t^{*}+\gamma^{2} l^{\top} \mathcal{A}(z(\bar{\varepsilon})) \bar{l}=a \gamma^{2}+2 b \gamma+c,
\end{aligned}
$$

where $c:=\left(t^{*}\right)^{\top} \mathcal{A}(z(\bar{\varepsilon})) t^{*}, b:=l^{\top} \mathcal{A}(z(\bar{\varepsilon})) t^{*}, a:=l^{\top} \mathcal{A}(z(\bar{\varepsilon})) l$.

By construction, for $\gamma^{*}:=1$ we have $w\left(\gamma^{*}\right)=t^{\top}(\bar{\varepsilon}) \mathcal{A}(z(\bar{\varepsilon})) t(\bar{\varepsilon})$ and it is the optimal value of the cost function of the problem (15) with $\varepsilon=\bar{\varepsilon}$. Hence

$$
w\left(\gamma^{*}\right)=\min _{\gamma \in\left[0, \gamma_{0}\right]} w(\gamma)=\min _{\gamma \in\left[0, \gamma_{0}\right]}\left(a \gamma^{2}+2 b \gamma+c\right) .
$$

Since $\gamma^{*}=1 \in\left(0, \gamma_{0}\right)$ in the formula above, then $2 a \gamma^{*}+2 b=2 a+2 b=0$. Therefore $-b=a$, which can be rewritten in the form $-l^{\top} \mathcal{A}(z(\bar{\varepsilon})) t^{*}=l^{\top} \mathcal{A}(z(\bar{\varepsilon})) l$, wherefrom we get

$$
(t(\bar{\varepsilon}))^{\top} \mathcal{A}(z(\bar{\varepsilon})) t^{*}=(t(\bar{\varepsilon}))^{\top} \mathcal{A}(z(\bar{\varepsilon})) t(\bar{\varepsilon}) .
$$

Since $t^{*} \in \operatorname{conv}\{\tau(i), i \in I\}$, then $t^{*}=\sum_{i \in I} \beta_{i} \tau(i), \quad \sum_{i \in I} \beta_{i}=1, \beta_{i} \geq 0, i \in I$.

Consequently, taking into account that $z(\bar{\varepsilon}) \in \mathcal{X}(\bar{\varepsilon})$ and $t(\bar{\varepsilon}) \geq 0$, we have

$$
(t(\bar{\varepsilon}))^{\top} \mathcal{A}(z(\bar{\varepsilon})) t^{*}=\sum_{i \in I} \beta_{i}(t(\bar{\varepsilon}))^{\top} \mathcal{A}(z(\bar{\varepsilon})) \tau(i) \geq 0 .
$$

But this inequality and inequality (14) contradict equality (16). The lemma is proved.

It should be noticed that the lemma above can be considered as a generalization of Lemma 2 from [13] since it is formulated for an arbitrary subset $\{\tau(i), i \in I\}$ of $T_{i m}$, while in Lemma 2 from [13] we considered the fixed subset of $T_{i m}$, namely, the set of vertices of conv $T_{i m}$.

\section{An extended dual problem for Linear Copositive Programming}

In this section, we will formulate an extended dual problem for problem (2).

Given an arbitrary cone $\mathcal{K} \in \mathcal{S}(p)$, the corresponding dual cone $\mathcal{K}^{*}$ is defined as

$$
\mathcal{K}^{*}:=\{A \in \mathcal{S}(p): A \bullet D \geq 0 \forall D \in \mathcal{K}\} .
$$

It is known that the cone of symmetric positive semidefinite matrices $\mathcal{P}(p)$ is self-dual, i.e. $\mathcal{P}^{*}(p)=\mathcal{P}(p)$ but the cone of symmetric copositive matrices $\mathcal{C} \mathcal{O} \mathcal{P}^{p}$ defined in (1), is not.

It can be shown (see e.g. [2]) that for the cone $\mathcal{C O} \mathcal{P}^{p}$, its dual cone $\mathcal{C P}^{p}$ is the cone of so-called completely positive matrices, $\mathcal{C} \mathcal{P}^{p}:=\operatorname{conv}\left\{x x^{\top}: x \in \mathbb{R}_{+}^{p}\right\}$, and $\mathcal{C P}^{p} \subset \mathcal{C O} \mathcal{P}^{p}$.

For a given finite integer $m_{0} \geq 0$, consider the following problem:

$$
\begin{array}{ll} 
& \max -\left(U+W_{m_{0}}\right) \bullet A_{0}, \\
\text { s.t. } \quad & \left(U_{m}+W_{m-1}\right) \bullet A_{j}=0, j=0,1, \ldots, n, m=1, \ldots, m_{0} ; \\
& \left(U+W_{m_{0}}\right) \bullet A_{j}=c_{j}, j=1,2, \ldots, n ; \\
& U \in \mathcal{C P}^{p}, W_{0}=\mathbb{O}_{p}, \\
& \left(\begin{array}{cc}
U_{m} & W_{m} \\
W_{m}^{\top} & D_{m}
\end{array}\right) \in \mathcal{C P}^{2 p}, m=1, \ldots, m_{0},
\end{array}
$$


where $U_{m} \in \mathcal{S}(p), D_{m} \in \mathcal{S}(p), W_{m} \in \mathbb{R}^{p \times p}, m=1, \ldots, m_{0}$, and $\mathbb{O}_{p}$ stays for the $p \times p$ null matrix.

Notice that in the case $m_{0}=0$, we consider that the index set $\left\{1, \ldots, m_{0}\right\}$ is empty and the constraints $\left(U_{m}+W_{m-1}\right) \bullet A_{j}=0, j=0,1, \ldots, n,\left(\begin{array}{cc}U_{m} & W_{m} \\ W_{m}^{\top} & D_{m}\end{array}\right) \in \mathcal{C P}^{2 p}, m=1, \ldots, m_{0}$, are absent in problem (17). Hence, for $m_{0}=0$, problem (17) takes the form

$$
\begin{aligned}
& \max -U \bullet A_{0}, \\
& \text { s.t. } \quad U \bullet A_{j}=c_{j}, j=1,2, \ldots, n ; \quad U \in \mathcal{C} \mathcal{P}^{p} .
\end{aligned}
$$

Lemma 2 [Weak duality] Let $x \in X$ be a feasible solution of the primal linear copositive problem (2) and

$$
\left(U_{m}, W_{m}, D_{m}, m=1, \ldots, m_{0} ; U\right)
$$

be a feasible solution of problem (17). Then the following inequality holds:

$$
c^{\top} x \geq-\left(U+W_{m_{0}}\right) \bullet A_{0} .
$$

Proof. For $m=1, \ldots, m_{0}$, it follows from the condition $\left(\begin{array}{cc}U_{m} & W_{m} \\ W_{m}^{\top} & D_{m}\end{array}\right) \in \mathcal{C} \mathcal{P}^{2 p}$ that there exists a matrix $B_{m}$ with non-negative elements in the form

$$
B_{m}=\left(\begin{array}{c}
V_{m} \\
L_{m}
\end{array}\right) \in \mathbb{R}^{2 p \times k(m)}
$$

such that

$$
\left(\begin{array}{cc}
U_{m} & W_{m} \\
W_{m}^{\top} & D_{m}
\end{array}\right)=B_{m} B_{m}^{\top}=\left(\begin{array}{c}
V_{m} \\
L_{m}
\end{array}\right)\left(\begin{array}{ll}
V_{m}^{\top} & L_{m}^{\top}
\end{array}\right) .
$$

The matrix $B_{m}$ above is composed by the blocks containing some matrices

$$
\begin{gathered}
V_{m}=\left(\tau^{m}(i), i \in I_{m}\right), L_{m}=\left(\lambda^{m}(i), i \in I_{m}\right), \\
\text { where } \tau^{m}(i) \in \mathbb{R}_{+}^{p}, \lambda^{m}(i) \in \mathbb{R}_{+}^{p}, i \in I_{m}, k(m):=\left|I_{m}\right|,
\end{gathered}
$$

Hence, for $m=1, \ldots, m_{0}$, the matrices $U_{m}, W_{m}, D_{m}$ in (17) admit representations

$$
U_{m}=V_{m} V_{m}^{\top}, \quad W_{m}=V_{m} L_{m}^{\top}, D_{m}=L_{m} L_{m}^{\top} .
$$

Consider the first group of constraints of the dual problem (17): $U_{1} \bullet A_{j}=0, j=0,1, \ldots, n$. Due to (21) and (22), these constraints can be rewritten in the form

$$
\sum_{i \in I_{1}}\left(\tau^{1}(i)\right)^{\top} A_{j} \tau^{1}(i)=0, j=0,1, \ldots, n
$$

It follows from (23) that for any $x \in \mathbb{R}^{n}$, we have

$$
\sum_{i \in I_{1}}\left(\tau^{1}(i)\right)^{\top} \mathcal{A}(x)\left(\tau^{1}(i)\right)=0 .
$$

Taking into account that for any $x \in X$, the inequalities

$$
t^{\top} \mathcal{A}(x) t \geq 0 \forall t \in \mathbb{R}_{+}^{p},
$$


should be fulfilled, equality (24) implies $\left(\tau^{1}(i)\right)^{\top} \mathcal{A}(x) \tau^{1}(i)=0, i \in I_{1}, \quad \forall x \in X$.

Thus one can conclude that $\tau^{1}(i) \in T_{i m}, i \in I_{1}$, and, consequently (see Proposition 2),

$$
\mathcal{A}(x) \tau^{1}(i) \geq 0, \quad i \in I_{1}, \forall x \in X .
$$

Suppose that for some $m \geq 1$, it was shown that

$$
\mathcal{A}(x) \tau^{m}(i) \geq 0, \quad i \in I_{m}, \forall x \in X .
$$

Due to (21) and (22), the constraints $\left(U_{m+1}+W_{m}\right) \bullet A_{j}=0, j=0,1, \ldots, n$, of problem (17) can be rewritten as follows:

$$
\sum_{i \in I_{m+1}}\left(\tau^{m+1}(i)\right)^{\top} A_{j} \tau^{m+1}(i)+\sum_{i \in I_{m}}\left(\lambda^{m}(i)\right)^{\top} A_{j} \tau^{m}(i)=0, j=0,1, \ldots, n .
$$

It follows from the latter equalities that for any $x \in \mathbb{R}^{n}$, we have

$$
\sum_{i \in I_{m+1}}\left(\tau^{m+1}(i)\right)^{\top} \mathcal{A}(x) \tau^{m+1}(i)+\sum_{i \in I_{m}}\left(\lambda^{m}(i)\right)^{\top} \mathcal{A}(x) \tau^{m}(i)=0 .
$$

By the hypothesis above, inequalities (27) are satisfied. Then, taking into account that $\lambda^{m}(i) \in$ $\mathbb{R}_{+}^{p}, i \in I_{m}$, and for any $x \in X$, inequalities (25) hold, we conclude from (28) that

$$
\left(\tau^{m+1}(i)\right)^{\top} \mathcal{A}(x) \tau^{m+1}(i)=0, \quad i \in I_{m+1}, \quad\left(\lambda^{m}(i)\right)^{\top} \mathcal{A}(x) \tau^{m}(i)=0, i \in I_{m}, \forall x \in X .
$$

Hence, $\tau^{m+1}(i) \in T_{i m}, i \in I_{m+1}$, and, according to Proposition 2, it holds

$$
\mathcal{A}(x) \tau^{m+1}(i) \geq 0, \quad i \in I_{m+1}, \forall x \in X .
$$

Now, replace $m$ by $m+1$ and repeat the considerations for all $m<m_{0}$.

Let $m=m_{0}$. In this case, relations (27) have the form

$$
\mathcal{A}(x) \tau^{m_{0}}(i) \geq 0, \quad i \in I_{m_{0}}, \quad \forall x \in X,
$$

and for $U=\sum_{i \in I} \tau(i) \tau^{\top}(i), \tau(i) \in \mathbb{R}_{+}^{p}, i \in I$, the constraints

$$
\left(U+W_{m_{0}}\right) \bullet A_{j}=c_{j}, \quad j=1, \ldots, n,
$$

of problem (17) can be represented as follows:

$$
\sum_{i \in I}(\tau(i))^{\top} A_{j} \tau(i)+\sum_{i \in I_{m_{0}}}\left(\lambda^{m_{0}}(i)\right)^{\top} A_{j} \tau^{m_{0}}(i)=c_{j}, j=1, \ldots, n .
$$

Then, evidently,

$$
\begin{aligned}
\sum_{j=1}^{n} c_{j} x_{j}= & \sum_{i \in I}(\tau(i))^{\top} \mathcal{A}(x) \tau(i)+\sum_{i \in I_{m_{0}}}\left(\lambda^{m_{0}}(i)\right)^{\top} \mathcal{A}(x) \tau^{m_{0}}(i) \\
& -\left(\sum_{i \in I}(\tau(i))^{\top} A_{0} \tau(i)+\sum_{i \in I_{m_{0}}}\left(\lambda^{m_{0}}(i)\right)^{\top} A_{0} \tau^{m_{0}}(i)\right) \\
& =\sum_{i \in I}(\tau(i))^{\top} \mathcal{A}(x) \tau(i)+\sum_{i \in I_{m_{0}}}\left(\lambda^{m_{0}}(i)\right)^{\top} \mathcal{A}(x) \tau^{m_{0}}(i)-\left(U+W_{m_{0}}\right) \bullet A_{0} .
\end{aligned}
$$

From (25) and (29) we conclude that

$$
(\tau(i))^{\top} \mathcal{A}(x) \tau(i) \geq 0, i \in I ; \quad\left(\lambda^{m_{0}}(i)\right)^{\top} \mathcal{A}(x) \tau^{m_{0}}(i) \geq 0, i \in I_{m_{0}}, \quad \forall x \in X .
$$

These inequalities together with equality (31) imply (20). The lemma is proved. 
Lemma 3 [Strong duality] Let problem (2) have an optimal solution. Then there exist a number $0 \leq m_{0}<\infty$ and a feasible solution

$$
\left(U_{m}^{0}, W_{m}^{0}, D_{m}^{0}, m=1, \ldots, m_{0} ; U^{0}\right)
$$

of problem (17) such that for any optimal solution $x^{0}$ of problem (2), it holds

$$
c^{\top} x^{0}=-\left(U^{0}+W_{m_{0}}^{0}\right) \bullet A_{0} .
$$

Proof. To prove the lemma, we will algorithmically construct the number $m_{0}$ and the matrices $(32)$.

Iteration \# 0. Consider the following SIP problem:

$$
\min _{(x, \mu)} \mu \text {, s.t. } t^{\top} \mathcal{A}(x) t+\mu \geq 0, t \in T,
$$

with the set $T$ defined in (5). If there exists a feasible solution $(\bar{x}, \bar{\mu})$ of this problem with $\bar{\mu}<0$, then set $m_{0}:=0$ and GO TO the Final step.

Otherwise for any $x \in X$, the vector $\left(x, \mu^{0}=0\right)$ is an optimal solution of problem (34). It should be noticed that in problem (34), the index set $T$ is a compact, and the constraints of this problem satisfy the Slater condition. Hence, (see e.g. [6]), there exist indices and numbers

$$
\tau(i) \in T, \quad \gamma(i)>0, i \in I_{1}, \quad 1 \leq\left|I_{1}\right| \leq n+1,
$$

such that

$$
\sum_{i \in I_{1}} \gamma(i)(\tau(i))^{\top} A_{j} \tau(i)=0, j=0,1, \ldots, n ; \quad \sum_{i \in I_{1}} \gamma(i)=1
$$

It follows from (35) that the set $I_{1}$ is nonempty and $\tau(i) \in T_{i m}, i \in I_{1}$.

Let us set $\beta_{1}(i):=\sqrt{\gamma(i)}, i \in I_{1}, V_{1}^{0}:=\left(\tau^{0}(i):=\beta_{1}(i) \tau(i), i \in I_{1}\right), U_{1}^{0}:=V_{1}^{0}\left(V_{1}^{0}\right)^{\top}$. Then equalities (35) take the form

$$
U_{1}^{0} \bullet A_{j}=0, j=0,1, \ldots, n .
$$

Denote $\mathcal{T}_{1}:=\operatorname{conv}\left\{\tau(i), i \in I_{1}\right\}$ and proceed to the next iteration.

Iteration \# 1. Consider the problem

$$
\begin{gathered}
\min _{(x, \mu)} \mu, \\
\text { s.t. } \mathcal{A}(x) \tau(i) \geq 0, i \in I_{1}, \quad t^{\top} \mathcal{A}(x) t+\mu \geq 0, t \in\left\{t \in T: \rho\left(t, \mathcal{T}_{1}\right) \geq \varepsilon_{1}\right\},
\end{gathered}
$$

where $\varepsilon_{1}>0$ is such a number that the set of feasible solutions of problem (37) with $\mu=0$ coincides with the set $X$ of feasible solutions of problem (2). According to Lemma 1, such $\varepsilon_{1}>0$ exists.

If there exists a feasible solution $(\bar{x}, \bar{\mu})$ of problem $(37)$ with $\bar{\mu}<0$, then STOP and GO TO the Final step with $m_{0}:=1$.

Otherwise, $\left(x, \mu^{0}=0\right)$ with any $x \in X$ is an optimal solution of problem (37). In the SIP problem (37), the index set $\left\{t \in T: \rho\left(t, \mathcal{T}_{1}\right) \geq \varepsilon_{1}\right\}$ is compact, and the constraints satisfy the following Slater type condition:

$$
\exists(\hat{x}, \hat{\mu}) \text { such that } \mathcal{A}(\hat{x}) \tau(i) \geq 0, i \in I_{1}, t^{\top} \mathcal{A}(\hat{x}) t+\hat{\mu}>0, t \in\left\{t \in T: \rho\left(t, \mathcal{T}_{1}\right) \geq \varepsilon_{1}\right\} .
$$


Hence (see [17]) there exist indices and numbers

$$
\tau(i) \in\left\{t \in T: \rho\left(t, \mathcal{T}_{1}\right) \geq \varepsilon_{1}\right\}, \gamma(i)>0, i \in \Delta I_{1}, \quad 1 \leq\left|\Delta I_{1}\right| \leq n+1,
$$

and vectors $\lambda^{1}(i) \in \mathbb{R}_{+}^{p}, i \in I_{1}$, such that

$$
\sum_{i \in \Delta I_{1}} \gamma(i)(\tau(i))^{\top} A_{j} \tau(i)+\sum_{i \in I_{1}}\left(\lambda^{1}(i)\right)^{\top} A_{j} \tau(i)=0, j=0,1, \ldots, n ; \quad \sum_{i \in \Delta I_{1}} \gamma(i)=1
$$

It follows from (39) that $\Delta I_{1} \neq \emptyset$ and for all $x \in X$, it holds

$$
(\tau(i))^{\top} \mathcal{A}(x) \tau(i)=0, i \in \Delta I_{1} ;\left(\lambda^{1}(i)\right)^{\top} \mathcal{A}(x) \tau(i)=0, i \in I_{1} .
$$

Hence $\tau(i) \in T_{i m}, i \in \Delta I_{1}$. From (35) and (39), we get

$$
\sum_{i \in I_{2}} \gamma(i)(\tau(i))^{\top} A_{j} \tau(i)+\sum_{i \in I_{1}}\left(\lambda^{1}(i)\right)^{\top} A_{j} \tau(i)=0, j=0,1, \ldots, n
$$

where $I_{2}:=I_{1} \cup \Delta I_{1}$. Let us apply to the data set

$$
\left\{\tau(i), \gamma(i), i \in \Delta I_{1} ; \quad \tau(i), \lambda^{1}(i), \gamma(i), i \in I_{1}\right\}
$$

a procedure which is described below.

Procedure DAM (Data Modification).

The Procedure starts with an initial data set

$$
\{\tau(i), \gamma(i), i \in \Delta I ; \quad \tau(i), \lambda(i), \gamma(i), i \in I\}
$$

such that

$$
\begin{gathered}
\tau(i) \in T_{i m}, \tau(i) \notin \operatorname{conv}\{\tau(i), i \in I\}, \gamma(i)>0, i \in \Delta I \\
\tau(i) \in T_{i m}, \lambda(i) \in \mathbb{R}_{+}^{p}, \gamma(i)>0, i \in I .
\end{gathered}
$$

Set $P_{+}(i):=\left\{k \in P: \tau_{k}(i)>0\right\}, i \in \Delta I \cup I$. If

$$
P_{+}(i) \cap\left(P \backslash P_{+}(s)\right) \neq \emptyset \forall s \in \Delta I \quad \forall i \in I,
$$

then STOP. The Procedure $\boldsymbol{D A} \boldsymbol{M}$ is complete.

If (43) is not satisfied, then find $s_{0} \in \Delta I$ and $i_{0} \in I$ such that

$$
P_{+}\left(i_{0}\right) \subset P_{+}\left(s_{0}\right) .
$$

Set $\theta:=\min _{k \in P} \theta_{k}>0$, where $\theta_{k}:=\left\{\begin{array}{l}\infty, \text { if } k \in P \backslash P_{+}\left(i_{0}\right), \\ \tau_{k}\left(s_{0}\right) / \tau_{k}\left(i_{0}\right), \text { if } k \in P_{+}\left(i_{0}\right) .\end{array}\right.$

Let us show that $\theta<1$. Suppose the contrary: $\theta \geq 1$. Hence $\theta_{k} \geq 1 \forall k \in P_{+}\left(i_{0}\right)$, and consequently, $\tau_{k}\left(s_{0}\right) \geq \tau_{k}\left(i_{0}\right)>0, k \in P_{+}\left(i_{0}\right)$. Notice that since

$$
1=\mathbf{e}^{\top} \tau\left(i_{0}\right)=\sum_{k \in P_{+}\left(i_{0}\right)} \tau_{k}\left(i_{0}\right) \leq \sum_{k \in P_{+}\left(i_{0}\right)} \tau_{k}\left(s_{0}\right) \leq \sum_{k \in P_{+}\left(s_{0}\right)} \tau_{k}\left(s_{0}\right)=\mathbf{e}^{\top} \tau\left(s_{0}\right)=1,
$$


we conclude that

$$
\sum_{k \in P_{+}\left(i_{0}\right)} \tau_{k}\left(s_{0}\right)=1, \sum_{k \in P_{+}\left(i_{0}\right)} \tau_{k}\left(i_{0}\right)=1, \text { and } \tau_{k}\left(s_{0}\right) \geq \tau_{k}\left(i_{0}\right)>0 \forall k \in P_{+}\left(i_{0}\right) .
$$

It follows from the latter conditions that $\tau\left(s_{0}\right)=\tau\left(i_{0}\right)$ which contradicts the assumption $\tau\left(s_{0}\right) \notin \operatorname{conv}\{\tau(i), i \in I\}$. The contradiction proves that $\theta<1$.

Since, by construction, $\theta$ is strictly positive, then the double inequality $0<\theta<1$ is valid.

In the data set (42), let us perform the following replacements:

$$
\begin{aligned}
& \tau\left(s_{0}\right) \longrightarrow \bar{\tau}\left(s_{0}\right)=\left(\tau\left(s_{0}\right)-\theta \tau\left(i_{0}\right)\right) /(1-\theta) \geq 0, \mathbf{e}^{\top} \bar{\tau}\left(s_{0}\right)=1, \bar{\tau}\left(s_{0}\right) \notin \operatorname{conv}\{\tau(i), i \in I\} \\
& \lambda\left(i_{0}\right) \longrightarrow \bar{\lambda}\left(i_{0}\right)=\lambda\left(i_{0}\right)+2 \gamma\left(s_{0}\right) \theta(1-\theta) \bar{\tau}\left(s_{0}\right) \geq 0 ; \\
& \gamma\left(i_{0}\right) \longrightarrow \bar{\gamma}\left(i_{0}\right)=\gamma\left(i_{0}\right)+\gamma\left(s_{0}\right) \theta^{2}>0 \\
& \gamma\left(s_{0}\right) \longrightarrow \bar{\gamma}\left(s_{0}\right)=\gamma\left(s_{0}\right)(1-\theta)^{2}>0
\end{aligned}
$$

All other data remain unchanged.

For the modified data set, check condition (43). If it is satisfied, then STOP, the procedure is complete. If (43) is not satisfied, then find new indices $s_{0} \in \Delta I$ and $i_{0} \in I$ such that inclusion (44) is valid and repeat the steps described above.

The Procedure $\boldsymbol{D A} \boldsymbol{M}$ is completely described.

Let us continue proving the Lemma. Recall that we are performing the Iteration \#1 of the algorithm. Having applied the Procedure DAM to the data set (41), one obtains a new (modified) data set in the same form (41) such that

- the indices $\tau(i), i \in I_{1}$, are the same as in the initial data set (i.e., the procedure leaved these indices unchanged);

- the modified indices $\tau(i), i \in \Delta I_{1}$, are the immobile ones in problem (3);

- for the modified indices $\tau(i)$ and numbers $\gamma(i), i \in \Delta I_{1}$, relations (38) are fulfilled;

- for the modified vectors $\lambda^{1}(i)$ and numbers $\gamma(i), i \in I_{1}$, it holds $\lambda^{1}(i) \in \mathbb{R}_{+}^{p}, \gamma(i)>0, i \in I_{1}$;

- for the modified data set (41), relations (43) with $\Delta I=\Delta I_{1}, I=I_{1}$ and (40) are satisfied.

Using the new data (obtained as the result of applying the Procedure DAM to the initial data set (41)), denote:

$$
\begin{gathered}
\beta_{2}(i):=\sqrt{\gamma(i)}, i \in I_{2}, V_{2}^{0}:=\left(\beta_{2}(i) \tau(i), i \in I_{2}\right), L_{1}^{0}:=\left(\lambda^{1}(i) / \beta_{1}(i), i \in I_{1}\right), \\
U_{2}^{0}:=V_{2}^{0}\left(V_{2}^{0}\right)^{\top}, W_{1}^{0}:=L_{1}^{0}\left(V_{1}^{0}\right)^{\top}, D_{1}^{0}:=L_{1}^{0}\left(L_{1}^{0}\right)^{\top} .
\end{gathered}
$$

Then relations (40) can be written as follows:

$$
\left(U_{2}^{0}+W_{1}^{0}\right) \bullet A_{j}=0, j=0,1, \ldots, n .
$$

GO TO the next iteration.

Iteration $\# m, m \geq 2$. By the beginning of the iteration, the numbers $\beta_{m}(i)>0, i \in I_{m}$, as well as the indices, vectors and numbers

$$
\tau(i) \in T_{i m}, \gamma(i)>0, i \in I_{m}=I_{m-1} \cup \Delta I_{m-1}, \lambda^{m-1}(i) \in \mathbb{R}_{+}^{p}, i \in I_{m-1},
$$


are found such that

- relations (43) with $\Delta I=\Delta I_{m-1} \neq \emptyset, I=I_{m-1}$ hold;

- the following equalities are satisfied:

$$
\sum_{i \in I_{m}} \gamma(i)(\tau(i))^{\top} A_{j} \tau(i)+\sum_{i \in I_{m-1}}\left(\lambda^{m-1}(i)\right)^{\top} A_{j} \tau(i)=0, j=0,1, \ldots, n .
$$

Using these data, matrix

$$
V_{m}^{0}=\left(\beta_{m}(i) \tau(i), i \in I_{m}\right) \quad \text { with } \beta_{m}(i)>0, \quad i \in I_{m},
$$

was constructed.

Denote $\mathcal{T}_{m}:=\operatorname{conv}\left\{\tau(i), i \in I_{m}\right\}$ and consider the problem

$$
\begin{gathered}
\min _{(x, \mu) \in \mathbb{R}^{n+1}} \mu, \\
\text { s.t. } \mathcal{A}(x) \tau(i) \geq 0, i \in I_{m}, \quad t^{\top} \mathcal{A}(x)+\mu \geq 0, t \in\left\{t \in T: \rho\left(t, \mathcal{T}_{m}\right) \geq \varepsilon_{m}\right\},
\end{gathered}
$$

where $\varepsilon_{m}>0$ is such a number that the feasible set of problem (48) with $\mu=0$ coincides with the feasible set $X$ of problem (2). According to Lemma 1, such $\varepsilon_{m}$ exists.

If there exists a feasible solution $(\bar{x}, \bar{\mu})$ of problem (48) with $\bar{\mu}<0$, then STOP and GO TO the Final step with $m_{0}:=m$.

Otherwise for any $x \in X$, vector $\left(x, \mu^{0}=0\right)$ is an optimal solution of problem (48).

Since in problem (48) the index set $\left\{t \in T: \rho\left(t, \mathcal{T}_{m}\right) \geq \varepsilon_{m}\right\}$ is compact, and the constraints satisfy the Slater type condition, then the optimality of $\left(x, \mu^{0}=0\right)$ provides that there exist indices and numbers

$$
\tau(i) \in \mathbb{R}_{+}^{p}, \mathbf{e}^{\top} \tau(i)=1, \tau(i) \notin \mathcal{T}_{m} ; \gamma(i)>0, i \in \Delta I_{m}, \quad 1 \leq\left|\Delta I_{m}\right| \leq n+1,
$$

and vectors

$$
\widehat{\lambda}^{m}(i) \in \mathbb{R}_{+}^{p}, i \in I_{m},
$$

that satisfy the following equalities:

$$
\sum_{i \in \Delta I_{m}} \gamma(i)(\tau(i))^{\top} A_{j} \tau(i)+\sum_{i \in I_{m}}\left(\widehat{\lambda}^{m}(i)\right)^{\top} A_{j} \tau(i)=0, j=0,1, \ldots, n ; \quad \sum_{i \in \Delta I_{m}} \gamma(i)=1
$$

It follows from (51) that

$$
(\tau(i))^{\top} \mathcal{A}(x) \tau(i)=0, i \in \Delta I_{m} ;\left(\widehat{\lambda}^{m}(i)\right)^{\top} \mathcal{A}(x) \tau(i)=0, i \in I_{m}, \quad \forall x \in X,
$$

and, therefore, $\tau(i) \in T_{i m}, i \in \Delta I_{m}$.

Based on (46) and (51), one can conclude that

$$
\sum_{i \in I_{m+1}} \gamma(i)(\tau(i))^{\top} A_{j} \tau(i)+\sum_{i \in I_{m}}\left(\lambda^{m}(i)\right)^{\top} A_{j} \tau(i)=0, j=0,1, \ldots, n
$$

where $I_{m+1}:=I_{m} \cup \Delta I_{m}$, and the vectors $\lambda^{m}(i) \in \mathbb{R}_{+}^{p}, i \in I_{m}$, are constructed as follows:

$$
\lambda^{m}(i)= \begin{cases}\lambda^{m-1}(i)+\widehat{\lambda}^{m}(i), & i \in I_{m-1}, \\ \widehat{\lambda}^{m}(i), & i \in \Delta I_{m-1}=I_{m} \backslash I_{m-1} .\end{cases}
$$


Having applied the described above Procedure DAM to the data set

$$
\left\{\tau(i), \gamma(i), i \in \Delta I_{m} ; \quad \tau(i), \lambda^{m}(i), \gamma(i), i \in I_{m}\right\},
$$

one will get the modified data set (in the same form) such that

- the indices $\tau(i), i \in I_{m}$, are the same as in the initial data set (these indices are not changed by the Procedure DAM);

- the modified indices $\tau(i), i \in \Delta I_{m}$, are the immobile ones in problem (3);

- for the modified indices $\tau(i)$ and numbers $\gamma(i), i \in \Delta I_{m}$, relations (49) are fulfilled;

- for the modified vectors $\lambda^{m}(i)$ and numbers $\gamma(i), i \in I_{m}$, it holds

$$
\lambda^{m}(i) \in \mathbb{R}_{+}^{p}, \gamma(i)>0, \quad i \in I_{m}
$$

- the modified data (53) satisfy relations (43) with $\Delta I=\Delta I_{m}, I=I_{m}$ and relations (52).

Using these new data, let us set

$$
\begin{gathered}
\beta_{m+1}(i):=\sqrt{\gamma(i)}, i \in I_{m+1} ; V_{m+1}^{0}:=\left(\beta_{m+1}(i) \tau(i), i \in I_{m+1}\right), L_{m}^{0}:=\left(\lambda^{m}(i) / \beta_{m}(i), i \in I_{m}\right), \\
U_{m+1}^{0}:=V_{m+1}^{0}\left(V_{m+1}^{0}\right)^{\top}, W_{m}^{0}:=L_{m}^{0}\left(V_{m}^{0}\right)^{\top}, D_{m}^{0}:=L_{m}^{0}\left(L_{m}^{0}\right)^{\top},
\end{gathered}
$$

where matrix $V_{m}^{0}$ was defined at the previous iteration according to (47). Then relations (52) can be written in the form:

$$
\left(U_{m+1}^{0}+W_{m}^{0}\right) \bullet A_{j}=0, j=0,1, \ldots, n .
$$

Perform the next Iteration \# $(m+1)$.

Final step. It will be proved in Lemma 4 (see below) that the algorithm consists of a finite number of iterations.

Hence, for some $0 \leq m_{0}<\infty$, one of the following situations will arise:

a) $m_{0}=0$ and for problem (34) there exists a feasible solution $(\bar{x}, \bar{\mu})$ with $\bar{\mu}<0$;

b) $m_{0}>0$ and for problem (48) with $m=m_{0}$ there exists a feasible solution $(\bar{x}, \bar{\mu})$ with $\bar{\mu}<0$.

In situation $a$ ) the constraints of the original problem (2) satisfy the Slater condition. Hence, according to the well-known optimality conditions (see [1], for example), if $x^{0}$ is an optimal solution of problem (2), then there exists a matrix $U^{0} \in \mathcal{C} \mathcal{P}^{p}$ such that

$$
U^{0} \bullet A_{j}=c_{j}, j=1,2, \ldots, n ; U^{0} \bullet \mathcal{A}\left(x^{0}\right)=0 .
$$

It follows from the relations above that $U^{0}$ is a feasible solution of the dual problem (18) and equality (33) holds.

Consider situation $b): m_{0}>0$. By the beginning of the final step, the matrices

$$
U_{s}^{0}, W_{s}^{0}, D_{s}^{0}, s=1, \ldots, m_{0}-1 ; W_{0}=\mathbb{O}_{p}, U_{m_{0}}^{0}=V_{m_{0}}^{0}\left(V_{m_{0}}^{0}\right)^{\top},
$$

the immobile indices $\tau(i)$, and numbers $\beta_{m_{0}}(i)>0, i \in I_{m_{0}}$, have been constructed.

Consider the problem

$$
\begin{gathered}
\min _{x} c^{\top} x, \\
\text { s.t. } \mathcal{A}(x) \tau(i) \geq 0, i \in I_{m_{0}}, t^{\top} \mathcal{A}(x) t \geq 0, t \in\left\{t \in T: \rho\left(t, \mathcal{T}_{m_{0}}\right) \geq \varepsilon_{m_{0}}\right\},
\end{gathered}
$$


where $\varepsilon_{m_{0}}>0$ is the number used when problem (48) with $m=m_{0}$ was formulated. The way the number $\varepsilon_{m_{0}}$ has been chosen guarantees that the feasible set of problem (55) coincides with the feasible set of problem (2).

Problem (55) satisfies a Slater type condition since, by construction,

$$
\mathcal{A}(\bar{x}) \tau(i) \geq 0, i \in I_{m_{0}}, t^{\top} \mathcal{A}(\bar{x}) t \geq-\bar{\mu}>0, t \in\left\{t \in T: \rho\left(t, \mathcal{T}_{m_{0}}\right) \geq \varepsilon_{m_{0}}\right\},
$$

and the index set $\left\{t \in T: \rho\left(t, \mathcal{T}_{m_{0}}\right) \geq \varepsilon_{m_{0}}\right\}$ is compact.

Let $x^{0}$ be an optimal solution of problem (2). Then vector $x^{0}$ is optimal in problem (55) as well. Hence, there exist indices, numbers and vectors

$$
\tau(i) \in \mathbb{R}_{+}^{p}, \mathbf{e}^{\top} \tau(i)=1, \tau(i) \notin \mathcal{T}_{m_{0}}, \gamma(i)>0, i \in I ; \lambda^{m_{0}}(i) \in \mathbb{R}_{+}^{p}, i \in I_{m_{0}},
$$

such that

$$
\begin{aligned}
& \sum_{i \in I} \gamma(i)(\tau(i))^{\top} A_{j} \tau(i)+\sum_{i \in I_{m_{0}}}\left(\lambda^{m_{0}}(i)\right)^{\top} A_{j} \tau(i)=c_{j}, j=1, \ldots, n, \\
& (\tau(i))^{\top} \mathcal{A}\left(x^{0}\right) \tau(i)=0, i \in I ;\left(\lambda^{m_{0}}(i)\right)^{\top} \mathcal{A}\left(x^{0}\right) \tau(i)=0, i \in I_{m_{0}} .
\end{aligned}
$$

Let us set

$$
\begin{aligned}
V^{0} & :=(\tau(i) \sqrt{\gamma(i)}, i \in I), \quad L_{m_{0}}^{0}:=\left(\lambda^{m_{0}}(i) / \beta_{m_{0}}(i), i \in I_{m_{0}}\right), \\
U^{0} & :=V^{0}\left(V^{0}\right)^{\top}, W_{m_{0}}^{0}:=L_{m_{0}}^{0}\left(V_{m_{0}}^{0}\right)^{\top}, D_{m_{0}}^{0}:=L_{m_{0}}^{0}\left(L_{m_{0}}^{0}\right)^{\top} .
\end{aligned}
$$

Then relations (56) take the form

$$
\left(U^{0}+W_{m_{0}}^{0}\right) \bullet A_{j}=c_{j}, j=1, \ldots, n .
$$

It follows from (36), (45), (54), and (58) that the constructed set of matrices (32) is a feasible solution of problem (17).

It was shown above that for any feasible solution $x \in X$ of problem (2) and any feasible solution (19) of problem (17), the inequality (20) holds. From the equalities (31) and (57), it follows that the feasible solution $x^{0} \in X$ of the primal problem (2) and the constructed above feasible solution (32) of problem (17) turn the inequality (20) into equality. The lemma is proved.

Lemma 4 The described in the proof of Lemma 3 algorithm is finite (i.e. it stops after a finite number of iterations).

Proof. If the algorithm has stopped on the Iteration \# 0 or the Iteration \# 1, the lemma is proved. Otherwise let us consider an Iteration \# $m$ of the algorithm for some $m \geq 2$.

At the beginning of this iteration, we have the set of indices $\tau(i) \in \mathbb{R}_{+}^{p}, i \in I_{m}$, where

$$
I_{m}=I_{m-1} \cup \Delta I_{m-1}=\Delta I_{0} \cup \Delta I_{1} \cup \ldots \cup \Delta I_{m-1} \text { and } \Delta I_{0}:=I_{1}, \Delta I_{s} \neq \emptyset, s=0, \ldots, m-1 \text {. }
$$

As before, denote $P_{+}(i):=\left\{k \in P: \tau_{k}(i)>0\right\}, i \in \Delta I_{s}, s=0,1, \ldots, m-1$.

Let $i_{s}$ be an index from the set $\Delta I_{s}: i_{s} \in \Delta I_{s}, \quad s=0,1, \ldots, m-1$.

For any $k, 2 \leq k \leq m$, and any $s, 0 \leq s \leq k-2$, by construction, it holds

$$
i_{s} \in I_{k-1}=\Delta I_{0} \cup \Delta I_{1} \cup \ldots \cup \Delta I_{k-2}, \quad i_{k-1} \in \Delta I_{k-1},
$$

and the relations (43) are fulfilled with $\Delta I=\Delta I_{k-1}$ and $I=I_{k-1}$. Hence

$$
P_{+}\left(i_{s}\right) \cap\left(P \backslash P_{+}\left(i_{k-1}\right)\right) \neq \emptyset, \quad s=0,1, \ldots, k-2, k=2, \ldots, m,
$$


wherefrom we conclude

$$
P_{+}\left(i_{s}\right) \not \subset P_{+}\left(i_{k-1}\right), \quad s=0,1, \ldots, k-2, k=2, \ldots, m .
$$

Consequently, all the sets $P_{+}\left(i_{s}\right), s=0,1, \ldots, m-1$, are different.

Taking into account that on each Iteration $\# m$ it holds $\Delta I_{s} \neq \emptyset, s=0,1, \ldots, m-1$, one can conclude that the number $m_{0}$ of the iterations fulfilled by the algorithm, cannot be greater than some finite number $m_{*}$, where $m_{*}$ is the maximal number of all different subsets of the set $P$ satisfying (59). The lemma is proved.

Remark 1 The main contribution of the algorithm used in the proof of Lemma 3, consists in the justification of the existence of a finite number $m_{0}$ and the corresponding feasible solution (32) of problem (17) for which equality (33) is satisfied.

It worth to mention that it was not the aim of this paper to find a "good "estimate of the minimal value of the number $m_{0}$.

Notice also that it is possible that someone can offer other (pehaps more complex) procedures for finding the finite sets of matrices (32) satisfying the constraints of problem (17) and the equality (33). Some of such procedures may provide a better (smaller than $m_{*}$ ) estimate of the number $m_{0}$.

Remark 2 In the case of isolated immobile indices, the set of immobile indices is finite: $T_{i m}=$ $\left\{t^{*}(j), j \in J_{*}\right\},\left|J_{*}\right|<\infty$ (see Proposition 2.5 in [14]). Then on each Iteration \# $m$ of the algorithm it holds

$$
\begin{gathered}
\Delta I_{m} \neq \emptyset,\left\{\tau(i), i \in \Delta I_{m}\right\} \subset\left\{t^{*}(j), j \in J_{*}\right\}, \\
\left\{\tau(i), i \in \Delta I_{k}\right\} \cap\left\{\tau(i), i \in \Delta I_{s}\right\}=\emptyset \forall k=1, \ldots, m \forall s=1, \ldots, m ; k \neq s,
\end{gathered}
$$

and relations (43) are satisfied. Hence is this case one does not need to use the Procedure $\boldsymbol{D A} \boldsymbol{M}$ and has $m_{0} \leq\left|J_{*}\right|$.

The main result of the paper can be formulated in the form of the following theorem which is a consequence of Lemmas 2 and 3 .

Theorem 1 There exists a finite $m_{0} \geq 0$ such that problem (17) is dual to the original linear copositive problem (2) and the strong duality relations are satisfied, i.e. if the primal problem (2) admits an optimal solution $x^{0}$, then the dual problem also has an optimal solution in the form (32) and equality (33) holds.

Remark 3 In our recent paper [13], we have suggested another strong dual formulation for Linear Copositive Programming. This formulation was based on the knowledge of the extremal points of the set conv $T_{i m}$. In the present paper, the extended dual problem for the linear copositive problem (2) is also obtained using the concept and the properties of the normalized immobile index set, but in its final formulation, we do not use neither the elements of this set (the immobile indices), nor the extremal points of its convex hull.

At the end of this section, we would like to note that as far as we know, with the exception of the mentioned above paper [13], all previously published optimal conditions and duality results for Linear Copositive Programming are formulated under the Slater condition. Here we do not suppose that the Slater condition is satisfied. All of this demonstrates the importance and novelty of the results of the paper. 


\section{Linear SDP}

Consider a linear SDP problem

$$
\min _{x} c^{\top} x \quad \text { s.t. } \mathcal{A}(x) \in \mathcal{P}(p) .
$$

Following [25], let us adduce the M. Ramana et al.' extended dual for this problem:

$$
\begin{gathered}
\max -\left(\widetilde{U}+\widetilde{W}_{m_{0}}\right) \bullet A_{0}, \\
\text { s.t. } \quad\left(\widetilde{U}_{m}+\widetilde{W}_{m-1}\right) \bullet A_{j}=0, j=0,1, \ldots, n, m=1, \ldots, m_{0}, \\
\left(\widetilde{E}+\widetilde{W}_{m_{0}}\right) \bullet A_{j}=c_{j}, j=1,2, \ldots, n ; \widetilde{U} \in \mathcal{P}(p), \widetilde{W}_{0}=\mathbb{O}_{p}, \\
\left(\begin{array}{cc}
\widetilde{U}_{m} & \widetilde{W}_{m} \\
\widetilde{W_{m}^{\top}} & I
\end{array}\right) \in \mathcal{P}(2 p), m=1, \ldots, m_{0} .
\end{gathered}
$$

It is easy to notice that the new dual problem (17) obtained in this paper for problem (2) has a similar structure and properties as the dual problem (ED-R) for SDP problem (60). Nevertheless, it is worth mentioning that these dual problems were obtained using different approaches: the dual problem (17) was formulated and its properties were established using (implicitly) the concept of the immobile indices while the dual SDP problem (ED-R) (referred in [25] as the regularized dual problem (DRP)) was derived using the notion of the minimal cone which was described there as the output of a special procedure.

To compare these results, let us apply the approach, developed in this paper for Linear Copositive Programming, to the SDP problem (60). Having repeated the described in Section 3 process of building the dual problem, one can obtain the extended dual to problem (60) in the form

$$
\begin{gathered}
\max -\left(U+W_{m_{0}}\right) \bullet A_{0}, \\
\text { s.t. } \quad\left(U_{m}+W_{m-1}\right) \bullet A_{j}=0, j=0,1, \ldots, n, m=1, \ldots, m_{0}, \\
\left(E+W_{m_{0}}\right) \bullet A_{j}=c_{j}, j=1,2, \ldots, n, U \in \mathcal{P}(p), \quad W_{0}=\mathbb{O}_{p}, \\
\left(\begin{array}{cc}
U_{m} & W_{m} \\
W_{m}^{\top} & D_{m}
\end{array}\right) \in \mathcal{P}(2 p), m=1, \ldots, m_{0} .
\end{gathered}
$$

The only difference in formulations (ED) and (ED-R) consists of the right lower blocks of the matrices (61) and (62). Let us show that these problems are equivalent.

In fact, let $\left(\widetilde{U}_{m}, \widetilde{W}_{m}, m=1, \ldots, m_{0}, \widetilde{U}\right)$ be a feasible solution of problem (ED-R). It is evident that $\left(U_{m}=\widetilde{U}_{m}, W_{m}=\widetilde{W}_{m}, D_{m}=I, m=1, \ldots, m_{0}, U=\widetilde{U}\right)$ is a feasible solution of problem (ED) with the same value of the cost function.

Now let us show that for any feasible solution

$$
\left(U_{m}, W_{m}, D_{m}, m=1, \ldots, m_{0}, U\right)
$$

of problem (ED) there exists a feasible solution

$$
\left(\widetilde{U}_{m}, \widetilde{W}_{m}, m=1, \ldots, m_{0}, \widetilde{U}\right)
$$

of problem (ED-R) with the same value of the cost function. 
Notice that for $m=1, \ldots, m_{0}$, it follows from the inclusion (62) that there exists a matrix

$$
B_{m}=\left(\begin{array}{c}
V_{m} \\
L_{m}
\end{array}\right) \in \mathbb{R}^{2 p \times k(m)} \text { with } V_{m} \in \mathbb{R}^{p \times k(m)} \text { and } L_{m} \in \mathbb{R}^{p \times k(m)},
$$

such that

$$
\left(\begin{array}{cc}
U_{m} & W_{m} \\
W_{m}^{\top} & D_{m}
\end{array}\right)=B_{m} B_{m}^{\top}=\left(\begin{array}{c}
V_{m} \\
L_{m}
\end{array}\right)\left(\begin{array}{ll}
V_{m}^{\top} & L_{m}^{\top}
\end{array}\right)
$$

Hence, for $m=1, \ldots, m_{0}$, matrices $U_{m}, W_{m}, D_{m}$ admit representations

$$
U_{m}=V_{m} V_{m}^{\top}, \quad W_{m}=V_{m} L_{m}^{\top}, D_{m}=L_{m} L_{m}^{\top},
$$

with some matrices $V_{m}, L_{m}$.

Let (63) be a feasible solution of problem (ED). Set

$$
\begin{gathered}
\widetilde{U}:=U, \widetilde{W}_{m_{0}}:=W_{m_{0}}, \quad \rho\left(m_{0}\right):=\max \left\{1, \mu_{\max }\left(L_{m_{0}}^{\top} L_{m_{0}}\right)\right\} \\
\widetilde{U}_{m_{0}}:=\rho\left(m_{0}\right) U_{m_{0}}, \widetilde{W}_{m_{0}-1}:=\rho\left(m_{0}\right) W_{m_{0}-1} .
\end{gathered}
$$

Here $\mu_{\max }(Q)$ denotes the maximal eigenvalue of matrix $Q \in \mathbb{R}^{p \times p}$. It is easy to check that, by construction, we have

$$
\left(\widetilde{U}+\widetilde{W}_{m_{0}}\right) \bullet A_{j}=c_{j}, j=1,2, \ldots, n ; \quad\left(\widetilde{U}_{m_{0}}+\widetilde{W}_{m_{0}-1}\right) \bullet A_{j}=0, j=0,1, \ldots, n .
$$

Let us show that

$$
\widetilde{U}_{m_{0}}-\widetilde{W}_{m_{0}} \widetilde{W}_{m_{0}}^{\top} \in \mathcal{P}(p)
$$

or equivalently,

$$
t^{\top} V_{m_{0}}\left(\rho\left(m_{0}\right) I-\widetilde{L}\left(m_{0}\right)\right) V_{m_{0}}^{\top} t \geq 0 \quad \forall t \in \mathbb{R}^{p}
$$

or

$$
\tau^{\top}\left(\rho\left(m_{0}\right) I-\widetilde{L}\left(m_{0}\right)\right) \tau \geq 0 \forall \tau \in\left\{\tau \in \mathbb{R}^{p}: \tau=V_{m_{0}}^{\top} t, t \in \mathbb{R}^{p}\right\} \subset \mathbb{R}^{p},
$$

where $\widetilde{L}\left(m_{0}\right):=L_{m_{0}}^{\top} L_{m_{0}}$.

It is known (see [20], p. 230) that for any real symmetric matrix $Q \in \mathcal{S}(p)$, the inequality $t^{\top} Q t \leq \mu_{\max }(Q) t^{\top} t$ is satisfied for any $t \in \mathbb{R}^{p}$. Hence

$$
\tau^{\top}\left(\rho\left(m_{0}\right) I-\widetilde{L}\left(m_{0}\right)\right) \tau=\rho\left(m_{0}\right) \tau^{\top} \tau-\tau^{\top} \widetilde{L}\left(m_{0}\right) \tau \geq\left(\rho\left(m_{0}\right)-\mu_{\max }\left(\widetilde{L}\left(m_{0}\right)\right)\right) \tau^{\top} \tau \geq 0 \forall \tau \in \mathbb{R}^{p} .
$$

Inclusion (65) is proved.

Suppose that for some $m \leq m_{0}$ we have constructed matrices

$$
\widetilde{U}, \widetilde{U}_{m_{0}}, \ldots, \widetilde{U}_{m}, \widetilde{W}_{m_{0}}, \ldots, \widetilde{W}_{m}, \widetilde{W}_{m-1},
$$

and such a number $\rho(m)>0$ that $\widetilde{W}_{m-1}=\rho(m) W_{m-1}$ and the following relations hold:

$$
\begin{gathered}
\left(\widetilde{U}+\widetilde{W}_{m_{0}}\right) \bullet A_{j}=c_{j}, j=1,2, \ldots, n \\
\left(\widetilde{U}_{s}+\widetilde{W}_{s-1}\right) \bullet A_{j}=0, j=0,1, \ldots, n, \widetilde{U}_{s}-\widetilde{W}_{s} \widetilde{W}_{s}^{\top} \in \mathcal{P}(p), \quad s=m_{0}, m_{0}-1, \ldots, m .
\end{gathered}
$$


Let us set

$$
\begin{gathered}
\rho(m-1):=\max \left\{1, \rho^{2}(m) \mu_{\max }\left(L_{m-1}^{\top} L_{m-1}\right)\right\}, \\
\widetilde{U}_{m-1}:=\rho(m-1) U_{m-1}, \quad \widetilde{W}_{m-2}:=\rho(m-1) W_{m-2} .
\end{gathered}
$$

Applying the described above rules for the cases where $m=m_{0}, m_{0}-1, \ldots, 2$, we can construct matrices $\widetilde{U}, \widetilde{U}_{m_{0}}, \ldots, \widetilde{U}_{2}, \tilde{W}_{m_{0}}, \ldots, \widetilde{W}_{2}, \widetilde{W}_{1}$ and the number $\rho(2)>0$ such that $\widetilde{W}_{1}=\rho(2) W_{1}$. Set $\rho(1):=\max \left\{1, \rho^{2}(2) \mu_{\max }\left(L_{1}^{\top} L_{1}\right)\right\}, \quad \widetilde{U}_{1}:=\rho(1) U_{1}$.

One can check that the constructed above matrices form a feasible solution (64) of problem (ED-R) and it holds

$$
\left(\widetilde{U}+\widetilde{W}_{m_{0}}\right) \bullet A_{0}=\left(U+W_{m_{0}}\right) \bullet A_{0}
$$

Hence, for the SDP problem (60), we have shown that the dual problem in the form (ED) is a slight modification of the known dual problem (ED-R).

Now, let us compare two pairs of primal and dual problems:

$(\alpha)$ the linear copositive problem (2) and its dual one (17), and

( $\beta$ ) the SDP problem (60) and its dual one (ED).

One can see that these pairs of dual problems are constructed in spaces $S(p)$ and $S(2 p)$ using the same rules, but their constraints are defined with the help of different dual cones:

- in the pair of problems (2) and (17), the cone $\mathcal{C O} \mathcal{P}^{p}$ is used to formulate the constraints of the primal copositive problem and the dual cones $\mathcal{C} \mathcal{P}^{p}$ and $\mathcal{C P}^{2 p}$ are used to formulate the constraints of the dual one;

- in the pair of SDP problems (60) and (ED), the cone $\mathcal{P}(p)$ is used to formulate the constraints of the primal SDP problem and the dual cones $\mathcal{P}^{*}(p)=\mathcal{P}(p)$ and $\mathcal{P}^{*}(2 p)=$ $\mathcal{P}(2 p)$ are used for the dual formulation.

This similarity points to a deep relationship between these two classes of conic problems, Linear Copositive Programming and SDP. At the same time, it is worth mentioning that copositive problems are more complex and less studied when compared with that of SDP.

Remark 4 When comparing the complexity of the mentioned above procedures of constructing the pairs of dual problems in SDP and Linear Copositive Programming, notice the following.

- For SDP problems, one has an estimate $m_{0} \leq \min \{n, p\}$ of the number $m_{0}$. This estimate can be found using the fact that the set of immobile indices for an SDP problem is a subspace of $\mathbb{R}^{p}$ and the properties of semi-definite matrices are well-studied [15].

- For linear copositive problems, determining a good estimate of $m_{0}$ is a much more challenging task as the set of immobile indices is a union of a finite number of convex cones in $\mathbb{R}^{p}$. Notice that the cone of copositive matrices and its dual cone (the cone of completely positive matrices) are not so well studied (there are many open questions here [5, 8]).

- The cones of copositive and completely positive matrices are neither self-dual nor homogeneous (see [9]).

As it was noticed above, finding a good estimate of the number $m_{0}$ for copositive problems was not our purpose here. We plan to devote a special paper to this issue. 


\section{Conclusions and future work}

The main contribution of the paper consists in developing a new approach to dual formulations in Linear Copositive Programming. This approach permitted us to formulate a new extended dual problem in explicit form and to close the duality gap between the optimal values of the copositive problem and its extended dual without any CQs or other additional assumptions.

To the best of our knowledge, with the exception of our previous papers [13, 16], in Linear Copositive Programming, there are no other known explicit strong dual formulations that do not require CQs.

In $[13,16]$, the dual problems were formulated based on the explicit knowledge of the immobile index set. The advantage of the dual results presented here if compare with that of the mentioned above results consists in the fact that now there is no need to find explicitly either the elements of the normalized immobile index set or the extremal points of its convex hull. For linear copositive problems, the dual formulations obtained in the paper are original and different from that published before.

The new dual formulation for Linear Copositive Programming is similar to the dual formulation for SDP problem proposed by M.Ramana et al. [25]. This similarity and the fact that the duality results obtained in this paper ( $i)$ do not use CQs, (ii) have explicit formulation, and (iii) are strong, motivate us to study other applications of the developed approach based the notion of the immobile indices.

In our future work, we are going to find a better estimate of the number $m_{0}$ that is essential for our dual formulation. To obtain this estimate, it will be necessary to study new properties of the extended dual problem and its feasible set. We plan also to apply the results of the paper for other classes of copositive problems with the aim to develop new explicit optimality conditions.

\section{References}

[1] Ahmed F., Dür M,, Still G., Copositive Programming via semi-infinite optimization, J. Optim. Theory Appl., 159, 322-340 (2013).

[2] Berman A., Shaked-Monderer N., Completely positive matrices, 216 p. World Scientific, New Jersey, London, Singapore, Hong Kong (2003).

[3] Baumer L.D., Extreme copositive quadratic forms. Pacific J. Math., 19(2), 197-204 (1966).

[4] Bomze I.M., Dür M., de Klerk E., Roos C., Quist A.J., Terlaky T., On Copositive Programming and standard quadratic optimization problems, Journal Global Optim., 18, 301-320 (2000).

[5] Bomze I.M., Copositive optimization - recent developments and applications, EJOR, 216(3), 509-520 (2012).

[6] Bonnans J.F., Shapiro A., Perturbation analysis of optimization problems, 601 p. SpringerVerlag, New-York (NY) 2000.

[7] Dickinson P.J.C., Hildebrand R., Considering copositivity locally. Journal of Mathematical Analysis and Applications, 437(2), 1184-1195 (2016). 
[8] Dür M., Copositive Programming - a Survey. In: Diehl M, Glineur F, Jarlebring E, Michielis W., editors. Recent advances in optimization and its applications in engineering, 535 p. Springer-Verlag, Berlin, Heidelberg (2010).

[9] Gowda M. S., Sznajder R., On the non-homogeneity of completely positive cones, Technical Report trGOW11-04, Department of Mathematics and Statistics University of Maryland Baltimore County Baltimore, MD 21250 USA November (2011) Available at http://www. optimization-online. org/DB HTML/2012/05/3464.html.

[10] Jeyakumar V., Lee G.M., Dihn N., New sequential Lagrange multiplier conditions characterizing optimality without constraint qualification for convex programs, SIAM J. Optim. Vol.14, No 2, (2003), pp.534-547.

[11] Kortanek K.O., Zhang Q., Perfect duality in semi-infinite and semidefinite programming. Math. Program., Ser. A 91: 127-144 (2001).

[12] Kostyukova O.I., Tchemisova TV., Optimality conditions for convex Semi-Infinite Programming problems with finitely representable compact index sets, J. Optim. Theory Appl., 175(1), 76-103 (2017).

[13] Kostyukova O.I., Tchemisova TV., Dudina O.S., Immobile indices and CQ-free optimality criteria for Linear Copositive Programming problems, Set-Valued Var.Anal., under revision.

[14] Kostyukova O.I., Tchemisova T.V., On a constructive approach to optimality conditions for convex SIP problems with polyhedral index sets, Optimization, 63(1), 67-91 (2014).

[15] Kostyukova O.I., Tchemisova T.V., Optimality criteria without constraint qualification for Linear Semidefinite Problems. Special Issue "Algebraic techniques in Graph Theory and Optimization", J. Math. Sci., 182(2), 126-143 (2012).

[16] Kostyukova O.I., Tchemisova T.V., Optimality conditions for Linear Copositive Programming problems with isolated immobile indices. Optimization. Published online: 15 Nov 2018 https://doi.org/10.1080/02331934.2018.1539482.

[17] Levin V.L., Application of E.Helly's theorem to convex programming, problems of best approximation and related questions. Math. USSR Sbornik, Vol. 8, No. 2 (1969) pp. 235247.

[18] Li S.J., Yang X.Q., and Teo K.L., Duality for semi-definite and semi-infinite programming. Optimization 52: 507-528 (2003).

[19] Luo Z.-Q., Sturm F.J., Zhang S., Duality results for conic convex programming. Econometric institute report no. 9719/a, Erasmus University Rotterdam, Erasmus School of Economics (ESE), Econometric Institute (1997).

[20] Magnus J. R., and Neudecker H., Matrix differential calculus with applications in statistics and econometrics. Third Edition John Wiley \& sons (2007).

[21] Motzkin T., Copositive quadratic forms. National Bureau of Standards Report 1818, pp. 11-12 (1952). 
[22] Pataki G., A simple derivation of a facial reduction algorithm and extended dual systems. Preprint available at http://www. unc. edu/ pataki/papers/fr.pdf (2000).

[23] Pólik I., Terlaky T., Exact duality for optimization over symmetric cones, AdvOL-Report No. 2007/10 McMaster University, Advanced Optimization Lab., Hamilton, Canada (2007).

[24] Ramana M.V., An exact duality theory for Semidefinite Programming and its complexity implications. DIMACS Technical report 95-02R, RUTCOR, Rutgers University, New Brunswick (NJ) (1995).

[25] Ramana M. V., Tuncel L., and Wolkowicz H., Strong duality for Semidefinite Programming. SIAM J. Optimization, vol. 7, N 3, pp. 641-662 (1997).

[26] Shapiro A., Duality, optimality conditions and perturbation analysis. In: Saigal R, Vandenberghe L, Wolkowicz H., editors. Semidefinite Programming and Applications Handbook, Kluwer Academic Publishers, Boston, 67-92 (2000).

[27] Tunçel L., Wolkowicz H., Strong duality and minimal representations for cone optimization, Comput. Optim. Appl., 53, 619-648 (2013).

[28] Weber G.-W., Generalized semi-infinite optimization and related topics, Heldermann publishing house, Research and Exposition in Mathematics 29, Lemgo, eds.: K.H. Hofmann and R. Willem, 2003. 